\title{
ESTACIONALIDAD REPRODUCTIVA EN PERRAS PASTOR ALEMÁN DE PEDIGRÍ EN LIMA METROPOLITANA
}

\author{
José Manuel Choy V. ${ }^{1}$ y Luisa Echevarría C. ${ }^{2}$
}

\section{Abstract}

\begin{abstract}
A study was done comparing the frecuency of estrus ocurrence in German Shepherd dogs in Lima, during the period 1997-2000. Data was obtained from the Peruvian German Shepherd Breeders Association, and from the local weather intitute (SENHAMI). The multiple lineal regresion was applied for datav analysis. A positive and significative relationship $(\mathrm{p}<0.05)$ between photoperiod, temperature, and month with the number of bitches in heat was found.
\end{abstract}

Key words: oestrus, estrous cycle, bitch, German Shepperd

\section{Resumen}

Se realizó un estudio retrospectivo de la frecuencia de presentación de estros en perras Pastor Alemán de pedigrí en Lima Metropolitana, que abarcó el periodo 19972000. Se trabajó con los registros de la Asociación Peruana de Propietarios de Perros Pastores Alemanes (APPPA) y los registros climatológicos del SENHAMI (temperatura ambiental y horas luz promedio). Se utilizó la Prueba de Regresión Lineal Múltiple para el análisis estadístico. Se encontró una asociación estadísticamente significativa $(\mathrm{p}<0.05)$ entre las variables fotoperiodo, temperatura y mes del año sobre el porcentaje de presentación de celos.

Palabras clave: estro, ciclo estral, perras, Pastor Alemán

\section{INTRODUCCIÓN}

La estacionalidad reproductiva de los perros es un fenómeno que se ha observado en varias partes del mundo. Las perras experimentan ciclos ováricos durante todo el año, aunque con mayor frecuencia al final del invierno y principio de la primavera.
La estacionalidad reproductiva en las perras se puede afectar por el tamaño, edad, condición corporal y enfermedades; así como por factores ambientales (temperatura, fotoperiodo y estrés) (Moon et al., 2000; Sánchez, 1999).

Las perras de talla pequeña, además de entrar más tempranamente a la puber-

\footnotetext{
${ }^{1}$ Práctica privada

${ }^{2}$ Laboratorio de Reproducción y Obstetricia Veterinaria, FMV-UNMSM

Dirección actual: Facultad de Veterinaria y Zootecnia, Universidad Peruana Cayetano Heredia

E-mail: luisa_ech@hotmail.com
} 
tad, tienen ciclos estrales de corta duración, por lo que tienen mayor probabilidad de tener mayor número de celos (Ortega et al., 2000). Asimismo, los perros criados en casas muestran poca o ninguna estacionalidad, mientras que el perro callejero puede retener cierta estacionalidad, muy similar a la de los cánidos silvestres. Por otro lado, la pérdida de la condición corporal extrema puede afectar la actividad reproductiva de la perra, y la mala condición corporal en forma constante puede bloquear la actividad estral por periodos prolongados.

Los registros del American Kennel Club para el Cocker, Setter, Gran Danés y Pequinés indican una mayor distribución uniforme de los estros a lo largo del año. Los Aireadles en perreras presentan una concentración de celos al final del verano y de menor frecuencia en el otoño; en tanto que el Basenji presenta sólo un estro por año, habitualmente en otoño (Feldman y Nelson, 2001).

En el caso de Lima, en un trabajo prospectivo se observó que aunque se presentaron celos en el transcurso de todo el año, se encontró una elevación de ocurrencia de celos en el verano y la primavera (Mariño, 1974).

Un parámetro importante en las perras es el denominado intervalo interestral, que tiene una duración prolongada y varía entre 5 a 10 meses (Sánchez, 1999). Por lo tanto, teniendo en cuenta que la perra posee un modelo reproductivo biológicamente diferente al resto de las especies, demanda gran interés el ampliar los aspectos endocrinos y clínicos de su ciclo estral. Además, se tiene información que tanto la temperatura como el fotoperiodo afecta la presentación de celos; aunque no se han realizado estudios de seguimiento por raza. Por todo lo expuesto, el presente estudio tiene como finalidad evaluar la frecuencia de presentación de celos de las perras Pastor Alemán y relacionarlos con los cambios climáticos de la ciudad de Lima.
Materiales y Métodos

Se seleccionó 300 hembras Pastor Alemán de pedigrí en Lima Metropolitana, con edades mayores de 18 meses y que se encontraban registradas en la Asociación Peruana de Criadores de Perros Pastores Alemanes (APPPA).

Se recolectó información correspondiente al periodo comprendido entre enero de 1997 hasta diciembre del 2000. Además, se obtuvo los promedios de temperatura media y horas luz de ese periodo (Servicio Nacional de Hidrología y Meteorología, SENHAMI). La información se analizó mediante la Prueba de Regresión Lineal Múltiple considerando como variable dependiente al porcentaje de celos y como variables independientes al mes del año, el año, el fotoperiodo y la temperatura ambiental.

\section{Resultados y Discusión}

La Fig. 1 muestra la frecuencia de presentación de celos en perras Pastor Alemán de pedigrí, donde se puede encontrar que entre los meses de marzo a julio se presentó el doble de ocurrencia de celos con relación a los observados en los meses de diciembre a febrero. Asimismo, se muestra la relación entre todas las variables de acuerdo al modelo de regresión, cuando interactúan en forma conjunta. Aquí se observa que en orden de importancia, las variables de mayor influencia sobre la presentación del celo son el fotoperiodo y la temperatura ambiental (temperatura media) $(\mathrm{p}<0.05)$, coincidiendo con lo reportado por Moon et al. (2000).

Se observó una correlación importante y significativa entre celo y fotoperiodo, así como entre fotoperiodo y temperatura. Se encontró que la correlación entre las variables aumenta a 0.617 indicando que existe una moderada correlación entre ellas. El $31.8 \%$ de la variación en el porcentaje de celos pudo 


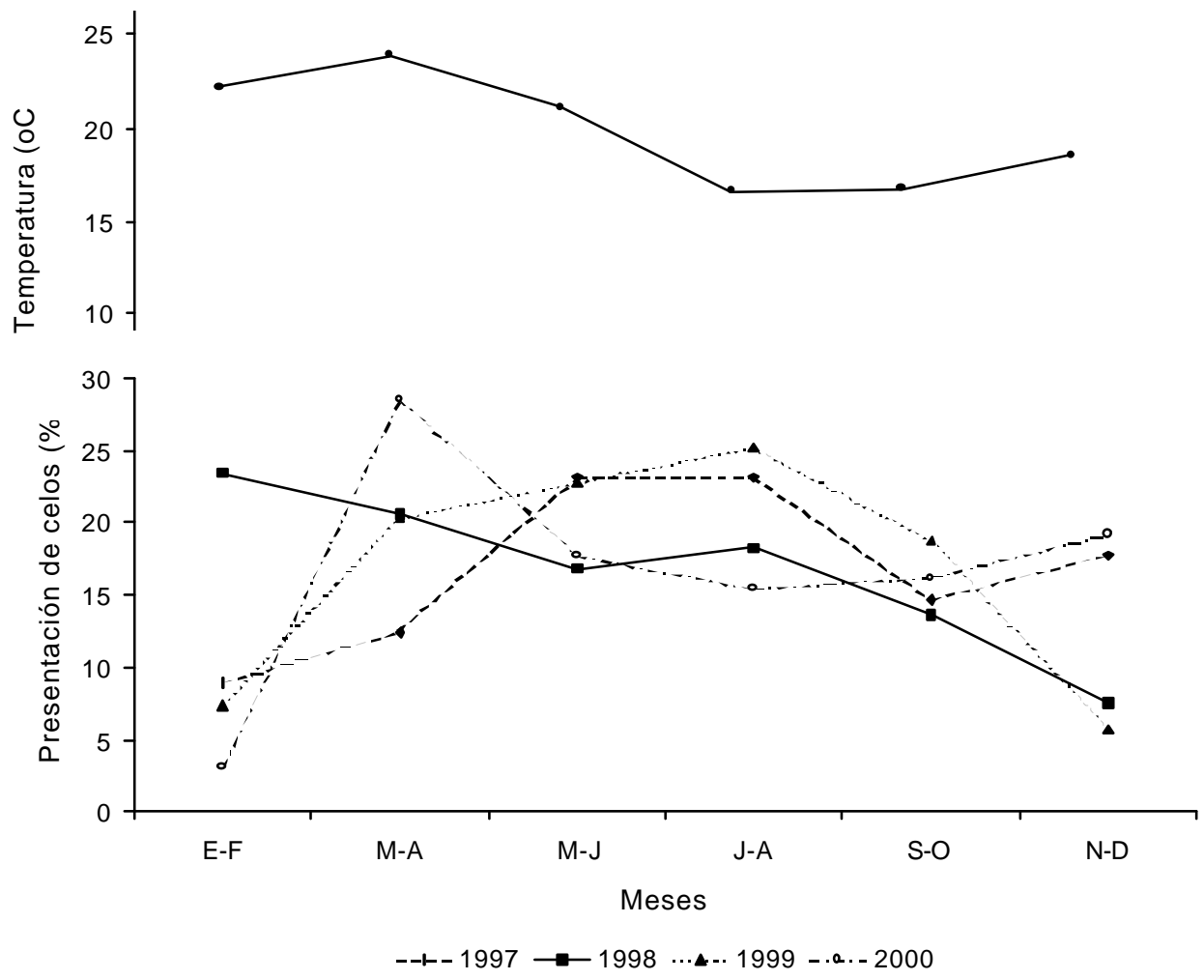

Figura 1. Porcentaje de presentación de celos comparado con temperatura y fotoperiodo, de perras Pastor Alemán desde el año 1997 al 2000 ( $n=740)$

Cuadro 1. Ocurrencia de celos en perras Pastor Alemán en el periodo 1997-2000

\begin{tabular}{|c|c|c|c|c|c|}
\hline \multirow{2}{*}{ Mes } & \multicolumn{4}{|c|}{ Año } & \multirow{2}{*}{ Total } \\
\hline & 1997 & 1998 & 1999 & 2000 & \\
\hline Enero & 6 & 21 & 2 & 3 & 42 \\
\hline Febrero & 14 & 29 & 7 & 1 & 57 \\
\hline Marzo & 13 & 17 & 16 & 17 & 79 \\
\hline Abril & 15 & 27 & 9 & 20 & 74 \\
\hline Mayo & 35 & 19 & 15 & 12 & 90 \\
\hline Junio & 17 & 17 & 13 & 11 & 61 \\
\hline Julio & 25 & 23 & 19 & 9 & 76 \\
\hline Agosto & 27 & 16 & 12 & 11 & 66 \\
\hline Septiembre & 18 & 17 & 8 & 13 & 56 \\
\hline Octubre & 15 & 12 & 15 & 8 & 50 \\
\hline Noviembre & 23 & 14 & 6 & 8 & 52 \\
\hline Diciembre & 17 & 2 & 1 & 17 & 37 \\
\hline Total & 225 & 214 & 123 & 130 & 740 \\
\hline
\end{tabular}


Cuadro 2. Temperatura promedio mensual desde los años 1997 al 2000 en Lima Metropolitana (SENHAMI, 2000)

\begin{tabular}{lllll}
\hline Mes & 1997 & 1998 & 1999 & 2000 \\
\hline Enero & 23.2 & 26.5 & 22.8 & 23.1 \\
Febrero & 24.3 & 27.3 & 24.9 & 23.7 \\
Marzo & 24.3 & 27.0 & 24.2 & 24.1 \\
Abril & 22.2 & 24.9 & 22.0 & 22.7 \\
Mayo & 21.8 & 19.1 & 21.5 & 19.4 \\
Junio & 22.0 & 17.5 & 17.2 & 16.8 \\
Julio & 21.8 & 16.2 & 14.9 & 16.3 \\
Agosto & 21.2 & 15.1 & 16.6 & 16.6 \\
Septiembre & 20.9 & 17.3 & 17.0 & 16.7 \\
Octubre & 20.5 & 16.0 & 18.4 & 18.2 \\
Noviembre & 22.2 & 17.3 & 19.1 & 18.7 \\
Diciembre & 24.6 & 19.7 & 20.8 & 21.2 \\
\hline
\end{tabular}

ser explicada por las variables mes, año, fotoperiodo y temperatura; por lo tanto, la ecuación de regresión es significativa $(\mathrm{p}<0.05)$.

\section{Conclusiones}

El fotoperiodo y la temperatura son las variables de mayor influencia en la presentación de celos en las perras pastores alemanes de pedigrí.

\section{Literatura Citada}

1. Feldman, D.V; R.W. Nelson. 2001. Endocrinología y reproducción en perros y gatos. $2^{a}$ ed. p 572-593. Ed. Mc GrawHill Interamericana. Mexico.
2. Mariño, J.V. 1974. Ciclo sexual en perras en los distritos de Bellavista, La Perla y La Punta, Comas, Independencia y San Martín de Porras en los Distritos de Lima y Callao. Tesis de Bachillerato. Facultad de Medicina Veterinaria, Univ. Nacional Mayor de San Marcos, Lima. $42 \mathrm{p}$.

3. Moon, P.F; H.N. Herb; J.W. Ludders; R.D. Gleed. 2000. Datos recientes en fisiología y endocrinología sexuales en la especie canina. J.A.A.H.A. 36 : $\mathrm{xx}-$ $\mathrm{xx}$.

4. Ortega-Pacheco, A.; J. RodríguezBuenfil; J.A. Leal-Ortega. 2000. Actividad estral de perras callejeras en la ciudad de Mérida, Yucatán y su relación con edad, tamaño y condición corporal. Rev. Biomed. 11:107-111. 